\title{
Correlation between XRCCI Arg399GIn genetic polymorphisms and susceptibility to bladder cancer: a meta-analysis
}

This article was published in the following Dove Press journal:

OncoTargets and Therapy

28 January 2016

Number of times this article has been viewed

\author{
Nannan Liu' \\ Xiawei $\mathrm{Fei}^{2}$ \\ Yi Shen' \\ Weifeng Shi' \\ Jinhong $\mathrm{Ma}^{\prime}$ \\ 'Department of Clinical Laboratory, \\ The Third Affiliated Hospital of \\ Suzhou University, Changzhou, \\ Jiangsu, People's Republic of China; \\ ${ }^{2}$ Department of Urology Surgery, \\ Qingpu Branch of Zhongshan Hospital \\ Affiliated to Fudan University, \\ Shanghai, People's Republic of China
}

Correspondence: Jinhong Ma Department of Clinical Laboratory, The Third Affiliated Hospital of Suzhou University, Changzhou 213003, Jiangsu, People's Republic of China Tel +8651968870895 Fax +865I98662 I235 Email mjhczyy@I26.com

\begin{abstract}
The relationship between $X R C C 1$ polymorphisms and bladder cancer has been widely studied. Here, our meta-analysis was conducted to evaluate the correlations between common genetic polymorphisms in XRCC1 and susceptibility to bladder cancer. In order to derive a more precise estimation of the association, 27 clinical case-control studies (which met all the inclusion criteria) were included in this meta-analysis. A total of 8,539 cancer cases and 10,750 controls were involved in this meta-analysis. Overall, no significant association was detected in allelic model (A allele vs T allele odds ratio $[\mathrm{OR}]=0.87,95 \%$ confidence interval $[\mathrm{CI}], 0.71-1.06$ ), homozygote comparison (AA vs GG OR $=1.12,95 \% \mathrm{CI}, 0.68-1.85$ ), heterozygote comparison (AT vs TT OR $=1.01,95 \%$ CI, 0.81-1.26), dominant model (AA + AG vs GG OR $=0.93,95 \%$ CI, 0.85-1.02), and recessive model (AA vs AG + GG OR $=1.01,95 \%$ CI, 0.88-1.15), but a moderately significant association was found for $\mathrm{AG}$ vs $\mathrm{GG}(\mathrm{OR}=0.241,95 \% \mathrm{CI}=0.17-0.35)$. Subgroup analysis based on ethnicity. Ethnicity analysis suggested that genetic polymorphisms in XRCC1 were not correlated with increased bladder cancer risk among Asians (all $P>0.05$ ). Therefore, we concluded that XRCC1 genetic polymorphism may not contribute to bladder cancer susceptibility in the present meta-analysis, and further well-designed studies with a large sample size are warranted to validate our conclusion.
\end{abstract}

Keywords: XRCC1, genetic polymorphism, susceptibility, bladder cancer, meta-analysis

\section{Introduction}

Bladder cancer is one of the most common health problems worldwide, the seventh most common malignancy in men, and 17 th most common in women. ${ }^{1}$ It is wellknown that the most common risk factors for bladder cancer include tobacco smoking, ${ }^{2}$ occupational exposure to chemicals, ${ }^{3}$ and schistosomiasis. ${ }^{1}$ Whereas, epidemiological studies have shown that genetic variants at one or more loci result in reduced DNA repair capacity and an increased cancer risk. ${ }^{4-6}$ In addition, a large number of single nucleotide polymorphisms in common DNA repair genes have also been identified and confirmed to be associated with several sporadic cancers. ${ }^{8,9}$

XRCC1 is located on chromosome $19 \mathrm{q} 13.2-13.3^{10,11}$ with a length of $33 \mathrm{~kb}$, and plays an essential role in DNA repair genes involved in base excision repair ${ }^{12}$ and single-strand breaks. ${ }^{13}$ To date, $\mathrm{XRCC} 1$ is the first cloned human gene associated with single-strand break repair ${ }^{14}$ and also related to sister-chromatid exchange. ${ }^{15}$ As previously described, there are three single nucleotide polymorphisms leads to amino acid substitutions in Arg194Trp in exon 6 (rs1799782), Arg280His in exon 9 (rs25489), and Arg399Gln in exon 10 (rs25487). ${ }^{16,17}$ 
Although several previous studies have evaluated the associations of XRCC1 polymorphisms with bladder cancer risk, the results are still inconsistent. In the present study, we performed a meta-analysis of all eligible studies to demonstrate the effect of XRCC1 Arg399Gln polymorphism on bladder cancer susceptibility.

\section{Materials and methods Identification of eligible studies}

PubMed, Embase, and Web of Science databases were searched in our meta-analysis. Case-control studies of the XRCC1 Arg399Gln polymorphism and bladder cancer susceptibility published before June 1, 2015 were included by using the keywords: "XRCC1", "X-ray repair crosscomplementing group 1", "Arg399Gln”, "polymorphism", "bladder cancer", and "urothelial carcinoma". The search was limited to English language papers. All reference lists of reviews and retrieved articles were manually screened for further potential studies.

\section{Inclusion and exclusion criteria}

The following criteria were used to determine inclusion eligibility: 1) a study that evaluated the correlation of XRCC1 Arg399Gln polymorphisms with bladder cancer susceptibility; 2) case-control study design; 3) full-text published articles; 4) a study that included sufficient genotype data for extraction. Furthermore, articles that did not meet our inclusion criteria were excluded.

\section{Data extraction}

Information was extracted carefully from all eligible publications independently and in duplicate by two authors. The following data were collected from each study: the first author's name, year of publication, country of origin, genotyping method, numbers of cases and controls, and evidence of Hardy-Weinberg equilibrium (HWE). The two authors reached consensus on each item.

\section{Statistical analysis}

The strength of association between the XRCC1 Arg399Gln polymorphism and bladder cancer was calculated by individual or pooled odds ratios (ORs) and $95 \%$ confidence intervals (CIs) using the STATA statistical software (Version 12.0, StataCorp LP, College Station, TX, USA). We evaluated the following comparisons to the XRCC1 Arg399Gln polymorphism including comparison of the variant allele with the wild-type allele (Gln allele vs Arg allele), the variant homozygote with the wild-type homozygote and the heterozygote (Gln/Gln vs Gln/Arg $+\mathrm{Arg} / \mathrm{Arg}$ ), the wild-type homozygote with the variant homozygote and the heterozygote (Arg/Arg vs Gln/Arg + Gln/Gln), and the variant homozygote with the heterozygote and wild-type homozygote (Gln/Gln vs Arg/Arg; Gln/Gln vs Gln/Arg). The statistical significance of the pooled ORs was assessed with the $\mathrm{Z}$ test and a $P$-value of $<0.05$ was considered significant. Chi-square-based $Q$ test was conducted to measure the heterogeneity between eligible studies, and the existence of heterogeneity was considered significant if $P<0.10 .^{11}$ When the between-study heterogeneity was absent, a fixed-effect model (the Mantel-Haenszel method) was used to pool the data from different studies. ${ }^{18}$ Otherwise, a random-effect model (the DerSimonian and Laird method) was applied. ${ }^{19}$ To explore the source of heterogeneity among variables such as ethnicity, and HWE status, both subgroup analyses and logistic met regression analyses were performed. ${ }^{20}$ Funnel plots and Egger's linear regression test were applied to investigate publication bias. ${ }^{21}$

\section{Results}

\section{Study selection and description}

A total of 27 eligible studies including 8,539 cases and 10,750 controls met the inclusion criteria. The HWE test was performed to determine the genotype distribution of the controls in all studies included. All of the studies, except for three, ${ }^{22-24}$ were not in HWE, and two studies ${ }^{25,26}$ lacked sufficient data for calculating the $P$-value to determine HWE.

\section{Quantitative data synthesis}

The study characteristics are summarized in Table 1. The genotype distribution and risk allele frequency of the included studies are summarized in Table 2. Overall, there was no significant correlation between the XRCC1 Arg399Gln polymorphism and bladder cancer risk for A allele vs $\mathrm{G}$ allele $(\mathrm{OR}=0.87,95 \% \mathrm{CI}=0.71-1.06, P=0.160$ for heterogeneity, Figure 1A), the codominant model AA vs $\mathrm{GG}(\mathrm{OR}=1.01,95 \% \mathrm{CI}=0.81-1.26, P=0.959$ for heterogeneity, Figure 1B), the dominant model AA/AG vs GG $(\mathrm{OR}=0.93,95 \% \mathrm{CI}=0.85-1.02, P=0.134$ for heterogeneity, Figure $1 \mathrm{C}$ ), and the recessive model AA vs $\mathrm{AG} / \mathrm{GG}$ $(\mathrm{OR}=1.01,95 \% \mathrm{CI}=0.88-1.15, P=0.934$ for heterogeneity, Figure 1D), but a moderately significant association was found for AG vs GG (OR $=0.241,95 \% \mathrm{CI}=0.17-0.35$, $P=0.000$ for heterogeneity, Figure 2). In subgroup analysis by ethnicity, no significant association was found between XRCC1 Arg399Gln polymorphism and bladder cancer risk among Asians $(P>0.05)$. 
Table I Baseline characteristics of studies included in the meta-analysis

\begin{tabular}{|c|c|c|c|c|c|}
\hline \multirow[t]{2}{*}{ Study } & \multirow[t]{2}{*}{ Year } & \multirow[t]{2}{*}{ Country } & \multirow[t]{2}{*}{ Method } & \multicolumn{2}{|c|}{ Number of subjects } \\
\hline & & & & Case & Controls \\
\hline Akhmadishina LZ et al $^{33}$ & 2014 & Russian & PCR-RFLP & 289 & 173 \\
\hline Chien-I Chiang $\mathrm{Cl}$ et $\mathrm{al}^{34}$ & 2014 & People's Republic of China & PCR-RFLP & 324 & 647 \\
\hline Volha $\mathrm{P}$ et $\mathrm{al}^{35}$ & 2014 & Belarus & PCR-RFLP & 332 & 364 \\
\hline Zhi Y et $\mathrm{al}^{36}$ & 2012 & People's Republic of China & PCR-RFLP & 302 & 311 \\
\hline Mittal RD et $\mathrm{al}^{37}$ & 2012 & India & ARMS PCR & 212 & 250 \\
\hline Gao $W$ et $\mathrm{al}^{38}$ & 2012 & USA & $\mathrm{PCR}+\mathrm{SSCP}$ & 192 & 313 \\
\hline Wang $M$ et al $^{39}$ & 2010 & People's Republic of China & PCR-RFLP & 234 & 253 \\
\hline Wen $\mathrm{H}$ et $\mathrm{al}^{26}$ & 2009 & People's Republic of China & TaqMan assay & 80 & 291 \\
\hline Mittal RD et $\mathrm{al}^{40}$ & 2008 & India & PCR-RFLP & 140 & 90 \\
\hline Fontana $\mathrm{L}$ et $\mathrm{al}^{41}$ & 2008 & France & TaqMan assay & 51 & 45 \\
\hline Covolo L et al ${ }^{42}$ & 2008 & Italy & PCR-RFLP & 197 & 211 \\
\hline Arizono $\mathrm{K}$ et $\mathrm{al}^{43}$ & 2008 & Japan & PCR-RFLP & 251 & 251 \\
\hline Andrew AS et $\mathrm{al}^{23}$ & 2008 & USA & PCR-RFLP & 990 & 1,253 \\
\hline Sak SC et al ${ }^{44}$ & 2007 & UK & TaqMan assay & 532 & 560 \\
\hline Huang $M$ et $\mathrm{al}^{25}$ & 2007 & USA & TaqMan assay & 613 & 696 \\
\hline Figueroa JD et $\mathrm{al}^{45}$ & 2007 & USA & TaqMan assay & $|, 06|$ & 996 \\
\hline Karahalil B et al ${ }^{46}$ & 2006 & Turkey & PCR-RFLP & 100 & 100 \\
\hline Andrew AS et $\mathrm{al}^{47}$ & 2006 & USA & PCR-RFLP & 306 & 538 \\
\hline Matullo $\mathrm{G}$ et $\mathrm{al}^{31}$ & 2006 & Italy & PCR-RFLP & 124 & 1,094 \\
\hline Wu $\times$ et $\mathrm{al}^{48}$ & 2006 & USA & TaqMan assay & 613 & 596 \\
\hline Matullo G et al $^{49}$ & 2005 & UK & PCR-RFLP & 311 & 312 \\
\hline Broberg $\mathrm{K}$ et $\mathrm{al}^{50}$ & 2005 & Sweden & Mass assay & 61 & 155 \\
\hline Kelsey KT et $\mathrm{al}^{24}$ & 2004 & USA & PCR-RFLP & 355 & 544 \\
\hline Sanyal S et $\mathrm{al}^{51}$ & 2004 & Sweden & PCR-RFLP & 311 & 246 \\
\hline Shen $M$ et $\mathrm{al}^{28}$ & 2003 & France & PCR-RFLP & 201 & 214 \\
\hline Matullo G et al $\left.\right|^{52}$ & 2001 & Italy & PCR-RFLP & 124 & 37 \\
\hline Stern $M C$ et $\mathrm{al}^{27}$ & 2001 & USA & PCR-RFLP & 233 & 210 \\
\hline
\end{tabular}

Abbreviations: PCR-RFLP, polymerase chain reaction-restriction fragment length polymorphism; AMRS PCR, amplification refractory mutation system polymerase chain reaction; PCR+SSCP, polymerase chain reaction and single-strand conformation polymorphism.

Table 2 Genotype distribution and risk allele frequency in all studies included

\begin{tabular}{|c|c|c|c|c|c|c|c|c|}
\hline \multirow[t]{2}{*}{ Study (year) } & \multicolumn{3}{|c|}{ Case } & \multicolumn{3}{|c|}{ Control } & \multicolumn{2}{|c|}{ HWE test } \\
\hline & GG & AG & AA & GG & AG & AA & $\chi^{2}$ & $P$-value \\
\hline Akhmadishina $\mathrm{LZ}$ et $\mathrm{al}^{33}$ & 86 & 143 & 60 & 60 & 88 & 25 & 0.639 & 0.424 \\
\hline Chien-I Chiang $\mathrm{Cl}$ et $\mathrm{al}^{34}$ & 179 & 108 & 37 & 350 & 253 & 44 & 0.036 & 0.850 \\
\hline Volha $\mathrm{P}$ et $\mathrm{al}^{35}$ & $|4|$ & 154 & 37 & $|5|$ & 165 & 48 & 0.076 & 0.782 \\
\hline Zhi Y et $\mathrm{al}^{36}$ & 121 & 151 & 30 & 148 & 143 & 20 & 3.571 & 0.588 \\
\hline Mittal RD et $\mathrm{al}^{37}$ & 67 & 106 & 39 & 102 & 109 & 39 & 1.186 & 0.276 \\
\hline Gao $W$ et $\mathrm{al}^{38}$ & 85 & 107 & - & 136 & 177 & - & - & - \\
\hline Wang $M$ et $\mathrm{al}^{39}$ & 113 & 102 & 19 & 105 & 126 & 22 & 3.414 & 0.065 \\
\hline Wen $\mathrm{H}$ et $\mathrm{al}^{26}$ & 46 & 34 & - & 153 & 138 & - & - & - \\
\hline Mittal RD et $\mathrm{al}^{40}$ & 37 & 76 & 27 & 73 & 81 & 36 & 2.459 & 0.117 \\
\hline Fontana $\mathrm{L}$ et $\mathrm{al}^{4 !}$ & 21 & 25 & 5 & 18 & 18 & 9 & 1.25 & 0.264 \\
\hline Covolo L et $\mathrm{al}^{42}$ & 92 & 105 & - & 91 & 120 & - & - & - \\
\hline Arizono $\mathrm{K}$ et $\mathrm{al}^{43}$ & 139 & 102 & 10 & 140 & 90 & 21 & 1.410 & 0.235 \\
\hline Andrew AS et $\mathrm{al}^{23}$ & 412 & 456 & 122 & 533 & 536 & 184 & 6.586 & 0.010 \\
\hline Sak SC et al ${ }^{44}$ & 218 & 248 & 66 & 226 & 259 & 75 & 0.003 & 0.953 \\
\hline Huang $M$ et $\mathrm{al}^{25}$ & 266 & 347 & - & 367 & 329 & - & - & - \\
\hline Figueroa JD et al ${ }^{45}$ & 434 & 494 & 133 & 433 & 453 & 110 & 0.273 & 0.602 \\
\hline Karahalil B et $\mathrm{al}^{46}$ & 49 & 38 & 13 & $4 I$ & 42 & 17 & 1.181 & 0.277 \\
\hline Andrew AS et al ${ }^{47}$ & 118 & 155 & 33 & 225 & 227 & 86 & 4.935 & 0.026 \\
\hline Matullo G et al $^{31}$ & 54 & 53 & 17 & 484 & 482 & 128 & 0.229 & 0.632 \\
\hline Wu $X$ et $a^{48}$ & 266 & 277 & 70 & 267 & 256 & 73 & 0.913 & 0.339 \\
\hline Matullo $\mathrm{G}$ et $\mathrm{al}^{49}$ & 136 & 135 & 40 & 120 & 145 & 47 & 0.087 & 0.768 \\
\hline Broberg $\mathrm{K}$ et $\mathrm{al}^{50}$ & 26 & 31 & 4 & 80 & 62 & 13 & $0.04 I$ & 0.840 \\
\hline Kelsey KT et $\mathrm{al}^{24}$ & 132 & 187 & 36 & 228 & 230 & 86 & 4.663 & 0.031 \\
\hline Sanyal S et $\mathrm{al}^{5 \mid}$ & 124 & 155 & 32 & 113 & 110 & 23 & 0.260 & 0.610 \\
\hline Shen $M$ et $\mathrm{al}^{28}$ & 93 & 87 & 21 & 92 & 98 & 24 & 0.168 & 0.682 \\
\hline Matullo $\mathrm{G}$ et al $^{52}$ & 53 & 58 & 13 & 12 & 19 & 6 & 0.111 & 0.739 \\
\hline Stern $M C$ et $\mathrm{al}^{27}$ & 96 & 116 & 21 & 88 & 96 & 26 & 0.000 & 0.982 \\
\hline
\end{tabular}

Abbreviation: HWE, Hardy-Weinberg equilibrium. 


\section{Sensitivity analysis}

The analysis of sensitivity was examined by sequential omission of individual studies. The significance of the pooled ORs in all individual and subgroup analyses was not excessively influenced by omitting any single study.

\section{Heterogeneity and publication bias}

Heterogeneity among studies was found in all comparisons of the XRCC1 Arg399Gln polymorphism. Therefore, the random effects model was used for single studies in the subgroup analysis to minimize the impact of bias. Funnel plots demonstrated

\begin{tabular}{|c|c|c|}
\hline Study (year) & OR $(95 \% \mathrm{Cl})$ & $\%$ weight \\
\hline Akhmadishina $\mathrm{LZ}$ et $\mathrm{al}^{33}$ & $0.79(0.61,1.04)$ & 5.07 \\
\hline Chien-I Chiang $\mathrm{Cl}$ et al ${ }^{34}$ & $0.60(0.50,0.73)$ & 5.33 \\
\hline Volha $\mathrm{P}$ et $\mathrm{al}^{35}$ & $1.04(0.84,1.29)$ & 5.24 \\
\hline Zhi Y et $\mathrm{al}^{36}$ & $0.79(0.62,1.01)$ & 5.17 \\
\hline Mittal RD et $\mathrm{al}^{37}$ & $0.77(0.59,1.01)$ & 5.10 \\
\hline Wang $\mathrm{M}$ et $\mathrm{al}^{39}$ & $1.09(0.84,1.42)$ & 5.09 \\
\hline Mittal RD et al ${ }^{40}$ & $0.77(0.56,1.04)$ & 4.92 \\
\hline Fontana L et al ${ }^{41}$ & $1.41(0.77,2.58)$ & 3.68 \\
\hline$A(2008)$ & $1.19(0.89,1.60)$ & 5.00 \\
\hline Sak SC et al ${ }^{44}$ & $1.02(0.86,1.22)$ & 5.37 \\
\hline Figueroa JD et al ${ }^{45}$ & $0.94(0.82,1.07)$ & 5.47 \\
\hline Matullo $\mathrm{G}$ et $\mathrm{al}^{31}$ & $0.67(0.43,1.04)$ & 4.39 \\
\hline Karahalil B et al ${ }^{46}$ & $0.23(0.18,0.28)$ & 5.30 \\
\hline Wu X et $\mathrm{al}^{48}$ & $1.01(0.85,1.20)$ & 5.38 \\
\hline Broberg $\mathrm{K}$ et $\mathrm{al}^{50}$ & $1.49(1.24,1.80)$ & 5.33 \\
\hline Matullo $\mathrm{G}$ et al ${ }^{49}$ & $0.55(0.36,0.82)$ & 4.53 \\
\hline Sanyal S et al ${ }^{51}$ & $0.89(0.68,1.15)$ & 5.10 \\
\hline Shen $\mathrm{M}$ et $\mathrm{al}^{28}$ & $0.98(0.73,1.31)$ & 5.00 \\
\hline Stern MC et al ${ }^{27}$ & $1.33(0.88,2.01)$ & 4.49 \\
\hline Matullo $G$ et al ${ }^{52}$ & $1.13(0.85,1.50)$ & 5.03 \\
\hline Overall $\left(I^{2}=92.6 \%, P=0.000\right)$ & $0.87(0.71,1.06)$ & 100 \\
\hline 0.185 & 5.42 & \\
\hline
\end{tabular}

\begin{tabular}{|c|c|c|}
\hline Study (year) & OR $(95 \% \mathrm{Cl})$ & $\%$ weight \\
\hline Akhmadishina LZ et al ${ }^{33}$ & $0.60(0.34,1.06)$ & 5.33 \\
\hline Chien-I Chiang Cl et al ${ }^{34}$ & $0.61(0.38,0.98)$ & 5.99 \\
\hline Volha $\mathrm{P}$ et al ${ }^{35}$ & $1.21(0.74,1.97)$ & 5.90 \\
\hline Zhi Y et al ${ }^{36}$ & $0.55(0.29,1.01)$ & 5.05 \\
\hline Mittal RD et al ${ }^{37}$ & $0.66(0.38,1.13)$ & 5.53 \\
\hline Wang $\mathrm{M}$ et $\mathrm{al}^{39}$ & $1.25(0.64,2.43)$ & 4.71 \\
\hline Mittal RD et al ${ }^{40}$ & $0.68(0.36,1.28)$ & 4.91 \\
\hline Fontana L et $\mathrm{al}^{41}$ & $-2.10(0.59,7.41)$ & 2.25 \\
\hline$A(2008)$ & $2.09(0.95,4.59)$ & 4.04 \\
\hline Sak SC et $\mathrm{al}^{44}$ & $1.10(0.75,1.60)$ & 6.63 \\
\hline Figueroa JD et al ${ }^{45}$ & $0.83(0.62,1.10)$ & 7.24 \\
\hline Matullo $\mathrm{G}$ et $\mathrm{al}^{31}$ & $0.54(0.22,1.36)$ & 3.43 \\
\hline Karahalil B et al ${ }^{46}$ & $0.84(0.47,1.50)$ & 5.28 \\
\hline Wu X et al ${ }^{48}$ & $1.04(0.72,1.50)$ & 6.70 \\
\hline Broberg $\mathrm{K}$ et $\mathrm{a}^{50}$ & $3.40(2.21,5.24)$ & 6.27 \\
\hline Matullo $\mathrm{G}$ et al ${ }^{49}$ & $1.06(0.32,3.52)$ & 2.41 \\
\hline Sanyal S et al ${ }^{51}$ & $0.79(0.44,1.43)$ & 5.18 \\
\hline Shen $M$ et $\mathrm{al}^{28}$ & $1.16(0.60,2.22)$ & 4.81 \\
\hline Stern $\mathrm{MC}$ et $\mathrm{al}^{27}$ & $1.58(0.64,3.89)$ & 3.50 \\
\hline Matullo $\mathrm{G}$ et al ${ }^{52}$ & $1.35(0.71,2.57)$ & 4.87 \\
\hline Overall $\left(I^{2}=67.2 \%, P=0.000\right)$ & $1.01(0.81,1.26)$ & 100 \\
\hline 0.135 & 41 & \\
\hline
\end{tabular}

Figure I (Continued) 


\section{C}

\begin{tabular}{|c|c|c|}
\hline Study (year) & OR $(95 \% \mathrm{Cl})$ & $\%$ weight \\
\hline Akhmadishina $L Z$ et $\mathrm{al}^{133}$ & $0.80(0.53,1.19)$ & 4.02 \\
\hline Chien-I Chiang Cl et $\mathrm{al}^{34}$ & $1.05(0.80,1.37)$ & 7.24 \\
\hline Volha $\mathrm{P}$ et $\mathrm{al}^{35}$ & $1.04(0.77,1.41)$ & 6.19 \\
\hline Zhi Y et al ${ }^{36}$ & $0.74(0.53,1.01)$ & 5.69 \\
\hline Mittal RD et $\mathrm{al}^{37}$ & $0.67(0.46,0.98)$ & 4.33 \\
\hline Wang $\mathrm{M}$ et $\mathrm{al}^{39}$ & $1.32(0.92,1.88)$ & 4.82 \\
\hline Mittal RD et al ${ }^{40}$ & $0.58(0.36,0.93)$ & 3.04 \\
\hline Fontana L et al ${ }^{41}$ & $1.05(0.46,2.38)$ & 1.15 \\
\hline$A(2008)$ & $0.98(0.69,1.40)$ & 4.94 \\
\hline Sak SC et al ${ }^{44}$ & $1.03(0.81,1.31)$ & 8.23 \\
\hline Figueroa JD et al ${ }^{45}$ & $0.90(0.76,1.07)$ & 11.43 \\
\hline Matullo $\mathrm{G}$ et $\mathrm{al}^{31}$ & $0.48(0.24,0.95)$ & 1.62 \\
\hline Karahalil B et al ${ }^{46}$ & $0.97(0.67,1.41)$ & 4.50 \\
\hline $\mathrm{Wu} X$ et $\mathrm{al}^{48}$ & $0.94(0.75,1.19)$ & 8.84 \\
\hline Broberg K et $a^{50}$ & $1.22(0.89,1.68)$ & 5.72 \\
\hline Matullo G et al ${ }^{49}$ & $0.70(0.38,1.27)$ & 2.05 \\
\hline Sanyal S et al ${ }^{51}$ & $0.78(0.56,1.09)$ & 5.25 \\
\hline Shen $\mathrm{M}$ et $\mathrm{al}^{28}$ & $1.14(0.78,1.68)$ & 4.27 \\
\hline Stern $\mathrm{MC}$ et $\mathrm{al}^{27}$ & $1.28(0.72,2.25)$ & 2.24 \\
\hline Matullo $\mathrm{G}$ et al ${ }^{52}$ & $0.97(0.67,1.42)$ & 4.43 \\
\hline Overall $\left(I^{2}=26.7 \%, P=0.132\right)$ & $0.93(0.85,1.02)$ & 100 \\
\hline 0.243 & 4.11 & \\
\hline
\end{tabular}

D

\begin{tabular}{|c|c|c|}
\hline Study (year) & OR (95\% Cl) & $\%$ weight \\
\hline Akhmadishina LZ et al ${ }^{33}$ & $1.55(0.93,2.58)$ & 5.26 \\
\hline Chien-I Chiang $\mathrm{Cl}$ et al ${ }^{34}$ & $1.77(1.12,2.80)$ & 6.15 \\
\hline Volha $\mathrm{P}$ et $\mathrm{al}^{35}$ & $0.83(0.52,1.30)$ & 6.19 \\
\hline Zhi $Y$ et $\mathrm{al}^{36}$ & $1.60(0.89,2.89)$ & 4.18 \\
\hline Mittal RD et $a^{37}$ & $1.22(0.75,1.99)$ & 5.64 \\
\hline Wang $M$ et $a^{39}$ & $0.93(0.49,1.76)$ & 3.64 \\
\hline Mittal RD et al ${ }^{40}$ & $1.02(0.59,1.78)$ & 4.61 \\
\hline Fontana L et al ${ }^{41}$ & $0.43(0.13,1.41)$ & 1.22 \\
\hline$A(2008)$ & $0.45(0.21,0.99)$ & 2.63 \\
\hline Sak SC et al ${ }^{44}$ & $0.92(0.64,1.31)$ & 8.73 \\
\hline Figueroa JD et al ${ }^{45}$ & $1.15(0.88,1.51)$ & 11.81 \\
\hline Matullo $\mathrm{G}$ et $\mathrm{al}^{31}$ & $0.82(0.38,1.78)$ & 2.60 \\
\hline Karahalil $B$ et $a^{46}$ & $1.20(0.70,2.07)$ & 4.76 \\
\hline Wu X et al ${ }^{48}$ & $0.92(0.65,1.31)$ & 8.89 \\
\hline Broberg K et al ${ }^{50}$ & $0.77(0.52,1.13)$ & 7.87 \\
\hline Matullo $\mathrm{G}$ et al ${ }^{49}$ & $0.77(0.24,2.45)$ & 1.25 \\
\hline Sanyal S et al ${ }^{51}$ & $1.11(0.63,1.95)$ & 4.49 \\
\hline Shen $\mathrm{M}$ et $\mathrm{al}^{28}$ & $0.92(0.50,1.72)$ & 3.85 \\
\hline Stern MC et $\mathrm{al}^{27}$ & $0.70(0.30,1.63)$ & 2.28 \\
\hline Matullo G et al ${ }^{52}$ & $0.70(0.38,1.29)$ & 3.97 \\
\hline Overall $\left(I^{2}=23.6 \%, P=0.165\right)$ & $1.01(0.88,1.15)$ & 100 \\
\hline 0.134 & 7.46 & \\
\hline
\end{tabular}

Figure I Odds ratios for associations between single nucleotide polymorphism Arg399Gln in XRCCl and bladder cancer risk.

Notes: (A) A allele vs G allele; (B) AA vs GG; (C) AA + AG vs GG; (D) AA vs AG + GG. Weights are from random effects analysis.

Abbreviations: $\mathrm{OR}$, odds ratio; $\mathrm{Cl}$, confidence interval. 


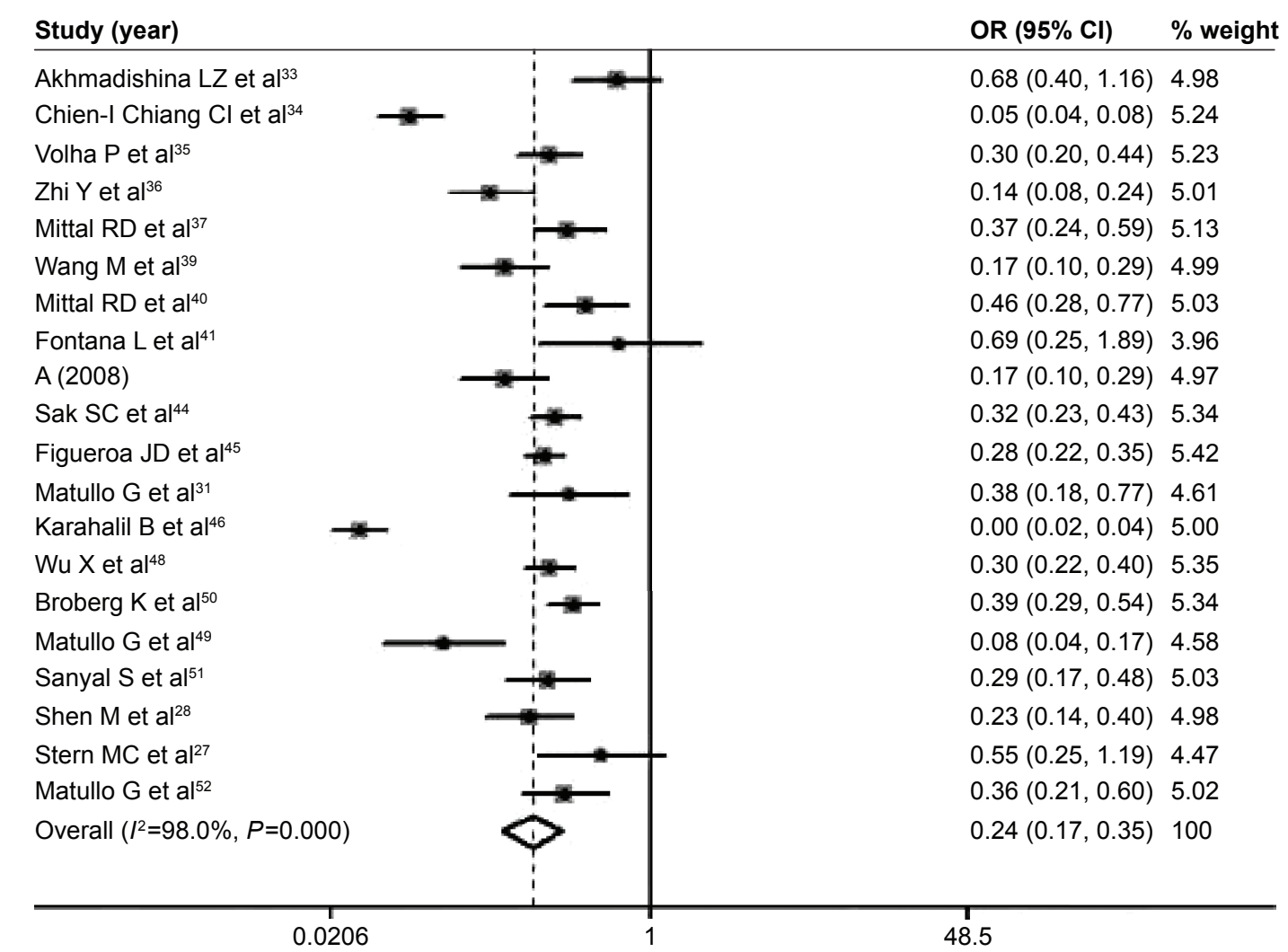

Figure 2 Forest plot of XRCCI Arg399GIn AG genotypes versus the GG genotype. Note: Weights are from random effects analysis.

Abbreviations: $\mathrm{OR}$, odds ratio; $\mathrm{Cl}$, confidence interval.

evidence of obvious asymmetry (Figure 3). Egger's test displayed strong statistical evidence of publication bias.

\section{Discussion}

Few studies have been conducted to investigate the association between the XRCC1 Arg399Gln polymorphism and bladder cancer risk in recent decades. Compared with those who had the Arg/Arg genotype, a slight decrease was found in risk for

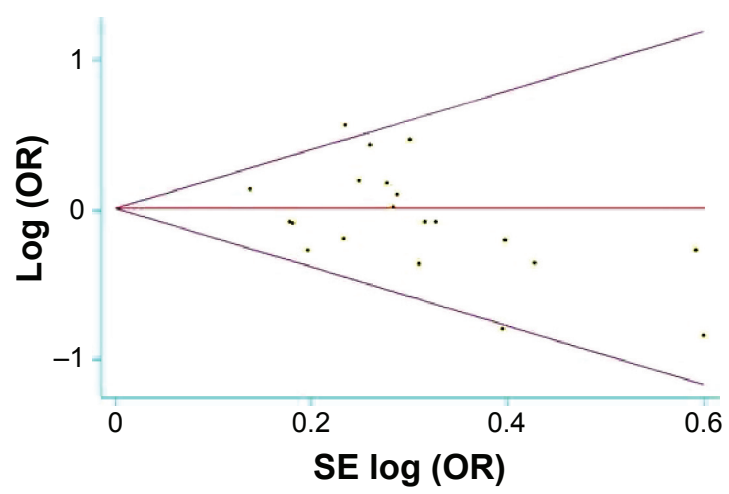

Figure 3 Funnel plot of two single nucleotide polymorphisms Arg399Gln in XRCCI and bladder cancer risk.

Note: Begg's funnel plot with pseudo $95 \%$ confidence limits. Abbreviations: OR, odds ratio; SE, standard error. individuals who carried the Gln/Gln genotype. ${ }^{27}$ Subsequently, a case-control investigation was carried out in Northern Italy, and the XRCC1 Arg399Gln polymorphism showed a protective effect on bladder cancer risk among heavy smokers. ${ }^{28} \mathrm{In}$ comparison with Gln allele vs Arg allele, (Gln/Gln + Gln/Arg) vs Arg/Arg, Gln/Gln vs (Gln/Arg + Arg/Arg), Gln/Gln vs Arg/ Arg, and Gln/Arg vs Arg/Arg, our meta-analysis based on these 27 studies revealed no correlation between the XRCC1 Arg399Gln polymorphism and bladder cancer risk.

As we know, mutations occurring in the nucleotide bases is the most common type of DNA damage, and they exhibit a high frequency (up to several thousand a day). Consequently, once the XRCC1 protein is lost, it may cause increased cell sensitivity to radiation, oxidative stress, and alkylating agents (eg, camptothecin). ${ }^{14}$ To date, more than 300 single nucleotide changes have been identified in the $\mathrm{XRCCl}$ gene. ${ }^{29}$ The Arg399Gln mutation leads to conformational changes in the XRCC1 protein that reduces its affinity for the multicomponent DNA repair protein complex. ${ }^{29}$

Presently, relationships between the XRCC1 Arg399Gln polymorphism and cancer development have been observed in several cancers. As previously reported, the alterations 
of XRCC1 are the most widely accepted suggestion to play a role in the pathogenesis of cancers. ${ }^{30,31}$ In particular, it has been found that the XRCC1 399Gln/Gln genotype was associated with lung cancer risk, as well as breast cancer risk in African Americans. ${ }^{32}$ However, no relationship between the XRCC1 Arg399Gln polymorphism and bladder cancer has been found in recent studies.

Notably, several limitations of our meta-analysis should be mentioned. Firstly, we strictly compiled data according to the rules of HWE, and ruled out three studies that might have caused the overall effects in our meta-analysis. Secondly, our systematic review was based on unadjusted data. Furthermore, the genotype information stratified for the main confounding variables was not available in the original papers.

Taken together, we have shown that there is no association between the XRCC1 Arg-399Gln polymorphism and bladder cancer risk. Additional large-scale studies with adequate methodological quality and controls for possible confounding effects should be conducted.

\section{Disclosure}

The authors declare that they have no conflicts of interest in this work.

\section{References}

1. Murta-Nascimento C, Schmitz-Drager BJ, Zeegers MP, et al. Epidemiology of urinary bladder cancer: from tumor development to patient's death. World J Urol. 2007;25(3):285-295.

2. Kogevinas M, 't Mannetje A, Cordier S, et al. Occupation and bladder cancer among men in Western Europe. Cancer Causes Control. 2003; 14(10):907-914.

3. Zeegers MP, Tan FE, Dorant E, van Den Brandt PA. The impact of characteristics of cigarette smoking on urinary tract cancer risk: a metaanalysis of epidemiologic studies. Cancer. 2000;89(3):630-639.

4. Helzlsouer KJ, Harris EL, Parshad R, Perry HR, Price FM, Sanford KK. DNA repair proficiency: potential susceptibility factor for breast cancer. J Natl Cancer Inst. 1996;88(11):754-755.

5. Wei Q, Spitz MR. The role of DNA repair capacity in susceptibility to lung cancer: a review. Cancer Metastasis Rev. 1997;16(3-4):295-307.

6. Sturgis EM, Castillo EJ, Li L, et al. Polymorphisms of DNA repair gene XRCC1 in squamous cell carcinoma of the head and neck. Carcinogenesis. 1999;20(11):2125-2129.

7. Miller MC 3rd, Mohrenweiser HW, Bell DA. Genetic variability in susceptibility and response to toxicants. Toxicol Lett. 2001;120(1-3): 269-280.

8. Alberg AJ, Jorgensen TJ, Ruczinski I, et al. DNA repair gene variants in relation to overall cancer risk: a population-based study. Carcinogenesis. 2013;34(1):86-92.

9. Kury S, Buecher B, Robiou-du-Pont S, et al. Low-penetrance alleles predisposing to sporadic colorectal cancers: a French case-controlled genetic association study. BMC Cancer. 2008;8:326.

10. Thompson LH, Bachinski LL, Stallings RL, et al. Complementation of repair gene mutations on the hemizygous chromosome 9 in $\mathrm{CHO}$ : a third repair gene on human chromosome 19. Genomics. 1989;5(4):670-679.

11. Mohrenweiser HW, Carrano AV, Fertitta A, et al. Refined mapping of the three DNA repair genes, ERCC1, ERCC2, and XRCC1, on human chromosome 19. Cytogenet Cell Genet. 1989;52(1-2):11-14.
12. Caldecott KW. XRCC1 and DNA strand break repair. DNA Repair (Amst). 2003;2(9):955-969.

13. Thompson LH, West MG. XRCC1 keeps DNA from getting stranded. Mutat Res. 2000;459(1):1-18.

14. Ladiges WC. Mouse models of XRCC1 DNA repair polymorphisms and cancer. Oncogene. 2006;25(11):1612-1619.

15. Wilson DM 3rd, Thompson LH. Molecular mechanisms of sisterchromatid exchange. Mutat Res. 2007;616(1-2):11-23.

16. Laczmanska I, Gil J, Karpinski P, et al. Influence of polymorphisms in xenobiotic-metabolizing genes and DNA-repair genes on diepoxybutane-induced SCE frequency. Environ Mol Mutagen. 2006; 47(9):666-673.

17. Hung RJ, Hall J, Brennan P, Boffetta P. Genetic polymorphisms in the base excision repair pathway and cancer risk: a HuGE review. Am J Epidemiol. 2005;162(10):925-942.

18. Poehlmann A, Roessner A. Importance of DNA damage checkpoints in the pathogenesis of human cancers. Pathol Res Pract. 2010; 206(9):591-601.

19. DerSimonian R, Laird N. Meta-analysis in clinical trials. Control Clin Trials. 1986;7(3):177-188.

20. Wang BS, Liu Z, Xu WX, Sun SL. CYP3A5*3 polymorphism and cancer risk: a meta-analysis and meta-regression. Tumour Biol. 2013; 34(4):2357-2366.

21. Peters JL, Sutton AJ, Jones DR, Abrams KR, Rushton L. Comparison of two methods to detect publication bias in meta-analysis. JAMA. 2006;295(6):676-680.

22. Andrew AS, Nelson HH, Kelsey KT, et al. Concordance of multiple analytical approaches demonstrates a complex relationship between DNA repair gene SNPs, smoking and bladder cancer susceptibility. Carcinogenesis. 2006;27(5):1030-1037.

23. Andrew AS, Karagas MR, Nelson $\mathrm{HH}$, et al. DNA repair polymorphisms modify bladder cancer risk: a multi-factor analytic strategy. Hum Hered. 2008;65(2):105-118.

24. Kelsey KT, Park S, Nelson HH, Karagas MR. A population-based case-control study of the XRCC1 Arg399Gln polymorphism and susceptibility to bladder cancer. Cancer Epidemiol Biomarkers Prev. 2004;13(8):1337-1341.

25. Huang M, Dinney CP, Lin X, Lin J, Grossman HB, Wu X. High-order interactions among genetic variants in DNA base excision repair pathway genes and smoking in bladder cancer susceptibility. Cancer Epidemiol Biomarkers Prev. 2007;16(1):84-91.

26. Wen H, Ding Q, Fang ZJ, Xia GW, Fang J. Population study of genetic polymorphisms and superficial bladder cancer risk in Han-Chinese smokers in Shanghai. Int Urol Nephrol. 2009;41(4):855-864.

27. Stern MC, Umbach DM, van Gils CH, Lunn RM, Taylor JA. DNA repair gene XRCC1 polymorphisms, smoking, and bladder cancer risk. Cancer Epidemiol Biomarkers Prev. 2001;10(2):125-131.

28. Shen M, Hung RJ, Brennan P, et al. Polymorphisms of the DNA repair genes XRCC1, XRCC3, XPD, interaction with environmental exposures, and bladder cancer risk in a case-control study in northern Italy. Cancer Epidemiol Biomarkers Prev. 2003;12(11 Pt 1):1234-1240.

29. Mao Y, Xu X, Lin Y, et al. Quantitative assessment of the associations between XRCC1 polymorphisms and bladder cancer risk. World J Surg Oncol. 2013;11:58.

30. Kiyohara C, Takayama K, Nakanishi Y. Association of genetic polymorphisms in the base excision repair pathway with lung cancer risk: a meta-analysis. Lung Cancer. 2006;54(3):267-283.

31. Matullo G, Dunning AM, Guarrera S, et al. DNA repair polymorphisms and cancer risk in non-smokers in a cohort study. Carcinogenesis. 2006; 27(5):997-1007.

32. Duell EJ, Wiencke JK, Cheng TJ, et al. Polymorphisms in the DNA repair genes XRCC1 and ERCC2 and biomarkers of DNA damage in human blood mononuclear cells. Carcinogenesis. 2000;21(5):965-971.

33. Akhmadishina LZ, Giliazova IR, Kutlyeva LR, et al. [DNA repair $\mathrm{XRCC1}$, XPD genes polymorphism as associated with the development of bladder cancer and renal cell carcinoma]. Genetika. 2014;50: $481-490$. 
34. Chien-I Chiang CI, Huang YL, Chen WJ, et al. XRCC1 Arg194Trp and Arg399Gln polymorphisms and arsenic methylation capacity are associated with urothelial carcinoma. Toxicol Appl Pharmacol. 2014;279:373-379.

35. Volha P, Ramaniuk VP, Nikitchenko NV, et al. Polymorphism of DNA repair genes OGG1, XRCC1, XPD and ERCC6 in bladder cancer in Belarus. Biomarkers. 2014;19:509-516.

36. Zhi Y, Yu J, Liu Y, et al. Interaction between polymorphisms of DNA repair genes significantly modulated bladder cancer risk. Int J Med Sci. 2012;9:498-505.

37. Mittal RD, Mandal RK, Gangwar R. Base excision repair pathway genes polymorphism in prostate and bladder cancer risk in North Indian population. Mech Ageing Dev. 2012;133:127-132.

38. Gao W, Romkes M, Zhong S, et al. Genetic polymorphisms in the DNA repair genes XPD and XRCC1, p53 gene mutations and bladder cancer risk. Oncol Rep. 2012;24:257-262.

39. Wang M, Qin C, Zhu J, et al. Genetic variants of XRCC1, APE1, and ADPRT genes and risk of bladder cancer. DNA Cell Biol. 2010;29:303-311

40. Mittal RD, Singh R, Manchanda PK, et al. May XRCC1 codon 399 mutant allele: a risk factor for recurrence of urothelial bladder carcinoma in patients on BCG immunotherapy. Cancer Biol Ther. 2008;7:645-650.

41. Fontana L, Bosviel R, Delort L, et al. DNA repair gene ERCC2, XPC, XRCC1, XRCC3 polymorphisms and associations with bladder cancer risk in a French cohort. Anticancer Res. 2008;28:1853-1856.

42. Covolo L, Placidi D, Gelatti U, et al. Bladder cancer, GSTs, NAT1, NAT2, SULT1A1, XRCC1, XRCC3, XPD genetic polymorphisms and coffee consumption: a case-control study. Eur J Epidemiol. 2008; $23: 355-362$
43. Arizono K, Osada Y, Kuroda Y. DNA repair gene hOGG1 codon 326 and XRCC1 codon 399 polymorphisms and bladder cancer risk in a Japanese population. Jpn J Clin Oncol. 2008;38:186-191.

44. Sak SC, Barrett JH, Paul AB, et al. DNA repair gene XRCC1 polymorphisms and bladder cancer risk. BMC Genet. 2007;8:13.

45. Figueroa JD, Malats N, Real FX, et al. Genetic variation in the base excision repair pathway and bladder cancer risk. Hum Genet. 2007;121:233-242.

46. Karahalil B, Kocabas NA, Ozçelik T. DNA repair gene polymorphisms and bladder cancer susceptibility in a Turkish population. Anticancer Res. 2006;26:4955-4958.

47. Andrew AS, Nelson HH, Kelsev KT, et al. Concordance of multiple analytical approaches demonstrates a complex relationship between DNA repair gene SNPs, smoking and bladder cancer susceptibility. Carcinogenesis. 2006;27:1030-1037.

48. Wu X, Gu J, Grossman HB, et al. Bladder cancer predisposition: a multigenic approach to DNA-repair and cell-cycle-control genes. $\mathrm{Am}$ J Hum Genet. 2006;78:464-479.

49. Matullo G, Guarrera S, Sacerdote C, et al. Polymorphisms/haplotypes in DNA repair genes and smoking: a bladder cancer case-control study. Cancer Epidemiol Biomarkers Prev. 2005;14:2569-2578.

50. Broberg K, Björk J, Paulsson K, et al. Constitutional short telomeres are strong genetic susceptibility markers for bladder cancer. Carcinogenesis. 2005;26:1263-1271.

51. Sanyal S, Festa F, Sakano S, et al. Polymorphisms in DNA repair and metabolic genes in bladder cancer. Carcinogenesis. 2004;25: 729-734.

52. Matullo G, Guarrera S, Carturan S, et al. DNA repair gene polymorphisms, bulky DNA adducts in white blood cells and bladder cancer in a case-control study. Int J Cancer. 2001;2:562-567.
OncoTargets and Therapy

\section{Publish your work in this journal}

OncoTargets and Therapy is an international, peer-reviewed, open access journal focusing on the pathological basis of all cancers, potential targets for therapy and treatment protocols employed to improve the management of cancer patients. The journal also focuses on the impact of management programs and new therapeutic agents and protocols on

\section{Dovepress}

patient perspectives such as quality of life, adherence and satisfaction The manuscript management system is completely online and includes a very quick and fair peer-review system, which is all easy to use. Visit http://www.dovepress.com/testimonials.php to read real quotes from published authors. 